\title{
CARACTERIZAÇÃO EPIDEMIOLÓGICA DOS ÓBITOS NO BRASIL POR MACRORREGIÃO DE 2016 A 2018
}

\author{
ARTIGO ORIGINAL \\ MELO, Carolina Simas ${ }^{1}$ \\ MACHADO, Daysane de Pinho ${ }^{2}$ \\ SANTOS, Ianna Karla Pereira dos ${ }^{3}$ \\ DIAS, Claudio Alberto Gellis de Mattos 4 \\ OLIVEIRA, Euzébio de ${ }^{5}$ \\ DENDASCK, Carla Viana ${ }^{6}$
}

FECURY, Amanda Alves ${ }^{7}$

MELO, Carolina Simas. Et al. Caracterização epidemiológica dos óbitos no Brasil por macrorregião de 2016 a 2018. Revista Científica Multidisciplinar Núcleo do Conhecimento. Ano 04, Ed. 12, Vol. 01, pp. 05-17. Dezembro de 2019. ISSN: 2448-

\footnotetext{
${ }^{1}$ Graduanda em Medicina, Universidade Federal do Amapá (UNIFAP).

${ }^{2}$ Nutricionista. Graduanda em Medicina, Universidade Federal do Amapá (UNIFAP).

${ }^{3}$ Graduanda em Medicina, Universidade Federal do Amapá (UNIFAP).

${ }^{4}$ Biólogo, Doutor em Teoria e Pesquisa do Comportamento, Professor e pesquisador do Colegiado de Química, Campus Macapá, Instituto Federal do Amapá (IFAP).

${ }^{5}$ Biólogo, Doutor em Doenças Topicais, Professor e pesquisador do Curso de Educação Física da Universidade Federal do Pará (UFPA).

${ }^{6}$ Teóloga, Doutora em Psicanálise, pesquisadora do Centro de Pesquisa e Estudos Avançados- CEPA.

${ }^{7}$ Biomédica, Doutora em Doenças Topicais, Professora e pesquisadora do Curso de Medicina do Campus Macapá, Universidade Federal do Amapá (UNIFAP).
} 
0959, Link de acesso: https://www.nucleodoconhecimento.com.br/saude/obitos-no$\underline{\text { brasil }}$

\section{RESUMO}

A Organização Mundial da Saúde (OMS) recomenda que as estatísticas de mortalidade sejam apresentadas pela causa de base do óbito, que é definida como doença ou injúria que iniciou a cascata de eventos que levam diretamente à morte, ou as circunstâncias de acidente ou violência que produziram ferimentos fatais. $O$ objetivo deste trabalho foi caracterizar epidemiologicamente o perfil dos casos registrados dos óbitos por macrorregião no Brasil, além de comparar os achados entre as macrorregiões, considerando os aspectos regionais, culturais e econômicos. Para a pesquisa foram utilizados dados secundários coletados no Departamento de Informática do Sistema Único de Saúde do Brasil (DATASUS), sobre causas de óbitos no período de 2016 a 2018. O total de casos de óbitos registrados no período do estudo foi de 1.488.868, com maior quantitativo na região Sudeste e o menor na região Norte. O sexo feminino apresentou número de óbitos maior que o masculino. Doenças do aparelho respiratório foram a principal causa e houve maior concentração de óbitos em indivíduos a partir de 60 anos de idade. Nas regiões Norte, Nordeste e CentroOeste os pardos foram os que mais morreram e, nas Regiões Sudeste e Sul, prevaleceram os brancos. Por meio do presente estudo, nota-se que o perfil da mortalidade brasileira tem sido modificado pela transição epidemiológica, apresentando quantitativos maiores nas Regiões mais desenvolvidas e associação com as DCNT, principalmente as doenças do aparelho respiratório. Além disso, é evidente a importância dos registros das informações sobre óbitos, da melhoria na coleta de dados e sistematização destes, com consequente melhor direcionamento e execução de políticas públicas brasileiras para prevenção e cuidados em saúde.

Palavras-chave: mortalidade, causa do óbito, Brasil, epidemiologia. 


\section{INTRODUÇÃO}

Para a consolidação do Sistema Único de Saúde (SUS), a gestão e desenvolvimento de Sistemas de Informação em Saúde (SIS) são um dos aspectos fundamentais. Estes devem proporcionar subsídios para qualificação dos processos de gestão do trabalho, além de possibilitar a reorganização das práticas nos sistemas de saúde. Diante disso, dada a importância do registro dos eventos vitais, em 1975 o Ministério da Saúde criou, no Brasil, o Sistema de Informações sobre Mortalidade (SIM) que, adotando o modelo padrão internacional da Declaração de Óbito (DO), realiza coleta contínua de dados sobre óbitos e suas causas (MOURA et al., 2016; FRANÇA et al., 2014). Tais estatísticas de mortalidade tornaram-se parte de um sistema informatizado a partir de 1979 e são usadas para definir condições de saúde e parâmetros socioeconômicos (ISHTANI et al., 2017; MARTINS, BUCHALLA, 2015).

A Organização Mundial da Saúde (OMS) recomenda que as estatísticas de mortalidade sejam apresentadas pela causa de base do óbito, que é definida como doença ou injúria que iniciou a cascata de eventos que levam diretamente à morte, ou as circunstâncias de acidente ou violência que produziram ferimentos fatais (MARTINS-MELO et al., 2012). A primeira classificação de doenças que se tornou de uso internacional, chamada de CID, foi criada em 1983 e vem sendo, desde este momento, revisada constantemente, sendo aprovada em 1989 a décima revisão (CID10). O aparato utilizado para atualização da mesma foi a criação do Grupo de Referência de Mortalidade (1997) e do Comitê de Revisão e Atualização (2000). O CID é de uso compulsório no Brasil para integrar a base de dados de códigos das informações sobre morbidade (Portaria 1311/GM de 12 de setembro de 1997) e também mortalidade (Portaria GM/MS n 1832/94, publicada no DOU n²18, de 03 de novembro de 1994) (LAURENTI et al., 2013).

A população brasileira vem passando nas últimas décadas por uma mudança no seu perfil populacional devido a transições demográfica, epidemiológica e tecnológica, o que vem refletindo em uma mudança também no perfil de mortalidade (BATISTA et al., 2018). Tais alterações estão em um conceito mais amplo, chamado de transição epidemiológica, associado às mudanças em longo prazo de alguns padrões (invalidez, 
mortalidade e morbidade) de uma população. Nesse processo de transição, três mudanças acontecem: as doenças não transmissíveis (DNT) e as causas externas substituem as transmissíveis, os mais idosos passam a receber a maior carga de morbimortalidade antes pertencente aos jovens e a mudança de uma situação em que a mortalidade era predominante para outra onde predomina a morbidade (CORRÊA, MIRANDA-RIBEIRO, 2017). Estima-se que de 2002 a 2030 haverá uma redução notável no perfil de óbitos no mundo e que entre 2010 e 2050, a proporção de idosos da população mundial duplique, passando de $8 \%$ para $16 \%$, sendo que esse crescimento será maior nos países em desenvolvimento (LEITE et al., 2015; OLIVEIRA-CAMPOS, CERQUEIRA, NETO, 2011).

Associando a mudança do perfil de doenças no Brasil com o envelhecimento populacional e a queda das taxas de fecundidade, estes fatores têm contribuído para a mudança no perfil epidemiológico, com a apresentação concomitante de doenças crônicas, doenças infecciosas e causas externas trazendo novos desafios ao sistema de saúde (MARINHO, PASSOS, FRANÇA, 2016; SOUZA et al., 2018). As doenças e agravos não transmissíveis são responsáveis por cerca de dois terços das doenças no Brasil e cerca de 55,2\% dos óbitos. Esse grupo é composto por agravos externos decorrentes de acidentes e violências, além das doenças crônicas não transmissíveis (DCNT) (OLIVEIRA-CAMPOS, CERQUEIRA, NETO, 2011).

As DCNT, caracterizadas, sobretudo pelas doenças cardiovasculares (DCV), neoplasias e doenças respiratórias crônicas, são, atualmente, as maiores causas de óbitos no Brasil e no mundo, sendo as DCV as responsáveis pela maioria dos casos (SIQUEIRA, SIQUEIRA-FILHO, LAND, 2017). Além disso, as DCNT são mais deflagradas em pessoas com baixa escolaridade e pouca renda, estando relacionadas à dificuldade de acesso à saúde e informação ou à exposição facilitada aos fatores de risco, exacerbando ainda mais as desigualdades no meio social (ALVES, MORAES NETO, 2015).

No Brasil há a existência da polarização entre os tipos de agravos à saúde (doenças infecto-parasitárias em oposição às doenças crônico-degenerativas) em associação com a polarização geográfica, definida como a existência de regiões dentro do 
hemisfério sul com taxa de mortalidade análoga a dos países mais carentes em contraposição com regiões com padrões de saúde semelhantes aos dos países desenvolvidos. Ademais, existe a polarização social que se traduz pela desproporção de morbidade e mortalidade entre a diversidade de grupos populacionais, existente em uma mesma cidade, estado ou região. Ela se sobressai por meio das discrepâncias de rendas, educação, moradia, alimentação, saneamento, além das diferenças de acesso aos serviços de saúde (DE ARAÚJO, 2012).

As mudanças sociais marcaram as duas últimas décadas no Brasil, especialmente pela diminuição da desigualdade e pobreza. Essa diminuição abrangeu o país como um todo, porém com um diferencial entre as regiões. A diminuição da desigualdade de renda entre 1990 e 2009 foi constatada em todas as regiões, entretanto foi maior no Sul e Sudeste e menor no Centro-Oeste. No mesmo período, a taxa de pobreza também reduziu e as regiões com maiores quedas foram em ordem decrescente: Sul, Centro-Oeste e Sudeste. Embora tenha havido essa redução, diferenças essenciais entre as regiões ainda existem, apresentando às regiões Norte e Nordeste as maiores taxas de pobreza (ANDRADE et al., 2013).

Ainda que o perfil das causas de óbitos pese sobre a expectativa de vida ao nascer, sua utilidade não sugere com precisão tal associação. Não obstante esse perfil seja um indicador de saúde razoável, ele não é capaz de traçar rumos adequados para promover melhoria das condições de saúde. Todavia, o modelo de mortalidade de uma determinada região, descrita como a mortalidade inerente a determinadas causas, possibilita que o grau de qualidade de vida real seja ponderado e proporciona aporte para políticas de saúde mais eficazes, ao passo que possibilita formar normas e objetivos prioritários (PAES, GOUVEIA, 2009).

\section{OBJETIVO}

Caracterizar epidemiologicamente o perfil dos casos registrados dos óbitos por macrorregião no Brasil, além de comparar os achados entre as macrorregiões. 


\section{MÉTODO}

Foi efetuado um estudo descritivo de caráter transversal e abordagem quantitativa, dispondo de dados secundários coletados no Departamento de Informática do Sistema Único de Saúde do Brasil (DATASUS), sobre causas de óbitos no período de 2016 a 2018, no seguinte endereço eletrônico www.datasus.gov.br/.

Na página do DATASUS, foram selecionados os (as) ícones/variáveis: Acesso a informação; TABNET; Epidemiológicas e Morbidade; Morbidade Hospitalar do SUS; Geral por Local de Internação, a partir de 2008; Brasil por região e Unidade de Federação. Para retirada das informações, foram escolhidos Linha: Região, Coluna: Não Ativa, Conteúdo: Óbitos, Período disponíveis: 2016 a 2018. Em seleções disponíveis, foram utilizadas as variáveis: Região, Capítulo CID-10, Faixa Etária 1, Sexo, Cor/Raça.

A faixa etária usada para a coleta dos dados foi a preconizada pela OMS, na qual crianças são consideradas de 0 a 9 anos completos, adolescentes de 10 a 19 anos completos, adultos de 20 a 59 anos completos e idosos, maiores de 60 anos.

Os dados colhidos foram sistematizados e tabulados em planilhas através do programa Microsoft Excel 2007, para pormenorização e elaboração de gráficos e tabelas.

\section{RESULTADOS}

O total de casos de óbitos registrados no Brasil, no período do estudo, foi de 1.488.868. A distribuição destes nas macrorregiões brasileiras pode ser observada no gráfico 1. Destaca-se a região Sudeste como a maior detentora do número de registros e a Norte como a menor. 
Gráfico 1. Quantitativo de óbitos registrados nos anos de 2016 a 2018, nas macrorregiões brasileiras.

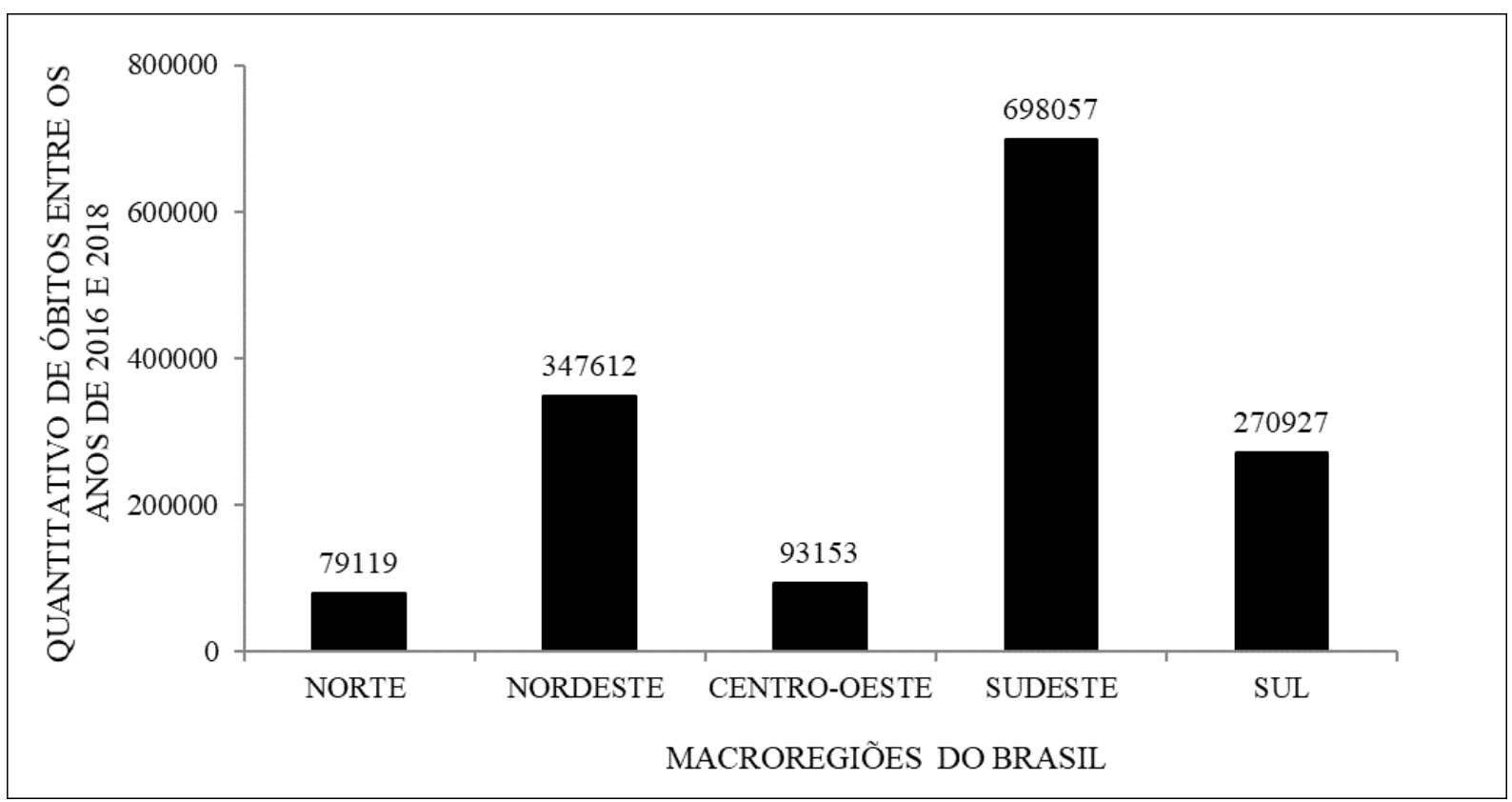

O gráfico 2 mostra o quantitativo de óbitos por sexo registrados nos anos de 2016 a 2018, nas macrorregiões brasileiras, considerando as cinco principais causas (doenças do aparelho respiratório; q doenças do aparelho circulatório, algumas doenças infecciosas e parasitárias, neoplasias e doenças do aparelho digestivo). Em todas as macrorregiões, o sexo feminino foi o que apresentou o maior quantitativo de óbitos. 
Gráfico 2. Quantitativo de óbitos por sexo registrados nos anos de 2016 a 2018, nas macrorregiões brasileiras.

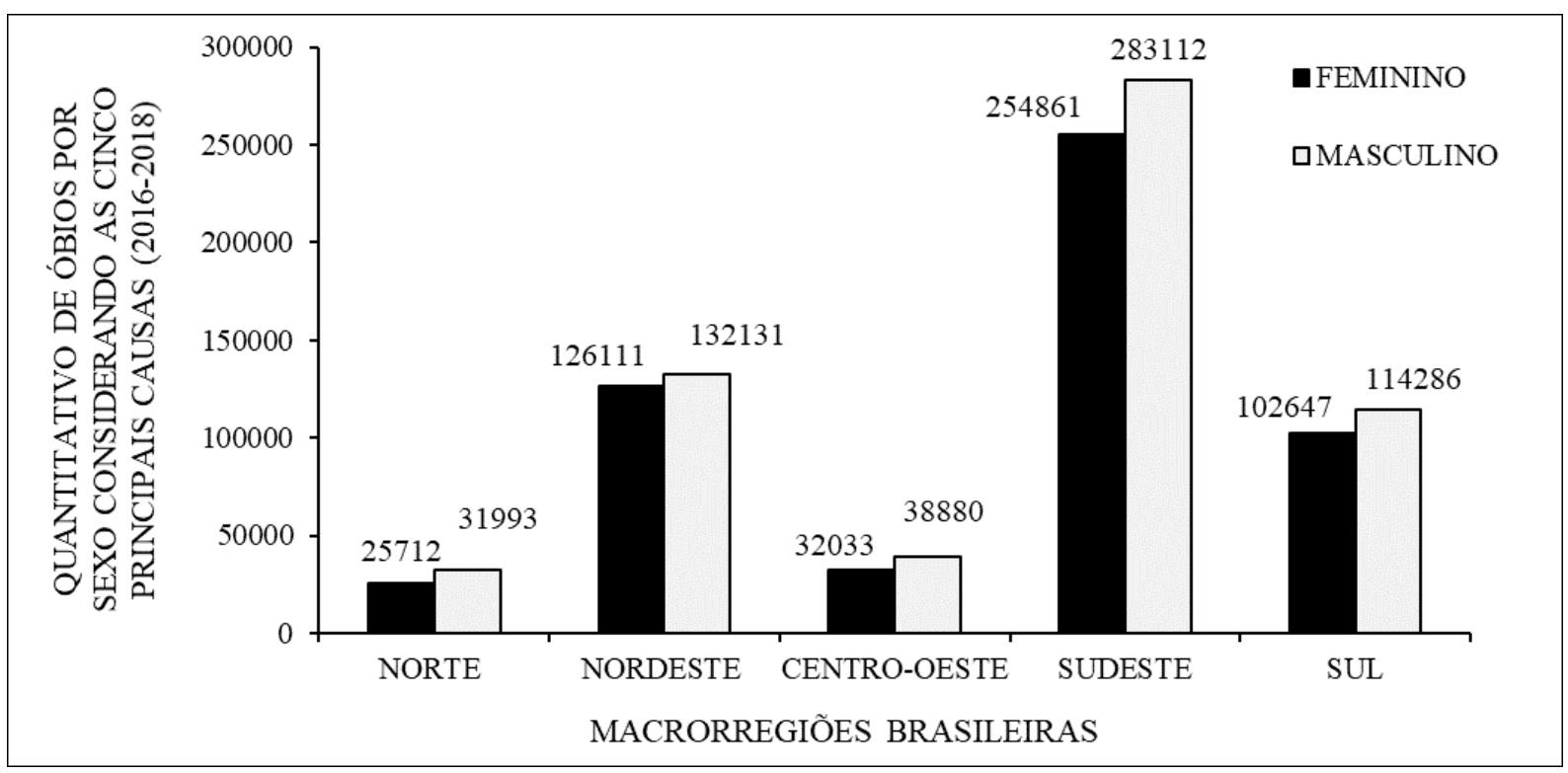

A tabela 1 mostra o quantitativo de óbitos pelas principais causas, relacionadas às macrorregiões brasileiras, registrados nos anos de 2016 a 2018. As doenças do aparelho respiratório aparecem como a principal causa de todos os óbitos $n$ as regiões Norte e Sul. Nas outras regiões, prevaleceram as do aparelho circulatório.

Tabela 1. Quantitativo de óbitos pelas principais causas, relacionadas às macrorregiões brasileiras, registrados nos anos de 2016 a 2018.

\begin{tabular}{|c|c|c|c|c|c|c|c|c|c|c|c|c|}
\hline & BRASIL & (\%) & NORTE & $(\%)$ & NORDESTE & $(\%)$ & $\begin{array}{l}\text { CENTRO- } \\
\text { OESTE }\end{array}$ & $(\%)$ & SUDESTE & (\%) & SUL & $(\%)$ \\
\hline $\begin{array}{l}\text { Doenças do aparelho } \\
\text { respiratório }\end{array}$ & 285281 & 24,99 & 15024 & 26,04 & 64032 & 24,80 & 18574 & 26,19 & 127612 & 23,72 & 60038 & 27,59 \\
\hline $\begin{array}{l}\text { Doenças do aparelho } \\
\text { circulatório }\end{array}$ & 282920 & 24,78 & 14747 & 25,56 & 67858 & 26,28 & 19372 & 27,32 & 130791 & 24,31 & 50791 & 23,34 \\
\hline $\begin{array}{lr}\text { Algumas } & \text { doenças } \\
\text { infecciosas } & \text { e } \\
\text { parasitárias } & \end{array}$ & 263953 & 23,12 & 13441 & 23,29 & 61250 & 23,72 & 13197 & 18,61 & 130618 & 24,28 & 45447 & 20,89 \\
\hline Neoplasias & 197850 & 17,33 & 8481 & 14,70 & 37684 & 14,59 & 12554 & 17,70 & 96418 & 17,92 & 42713 & 19,63 \\
\hline $\begin{array}{l}\text { Doenças do aparelho } \\
\text { digestivo }\end{array}$ & 111762 & 9,79 & 6012 & 10,42 & 27418 & 10,62 & 7215 & 10,17 & 52534 & 9,77 & 18583 & 8,54 \\
\hline TOTAL & 1141766 & 100,00 & 57705 & 100,00 & 258242 & 100,00 & 70912 & 100,00 & 537973 & 100,00 & 217572 & 100,00 \\
\hline
\end{tabular}

$\mathrm{Na}$ tabela 2 é demonstrado o quantitativo de óbitos pelas principais causas, relacionadas ao sexo, registrados nos anos de 2016 a 2018. Doenças do aparelho 
circulatório foram as principais causas para o sexo feminino, enquanto que, para o masculino, prevaleceram as do aparelho respiratório.

Tabela 2. Quantitativo de óbitos pelas principais causas, relacionadas ao sexo, registrados nos anos de 2016 a 2018.

\begin{tabular}{lllll} 
& FEMININO & $(\%)$ & MASCULINO & $(\%)$ \\
\hline Doenças do aparelho respiratório & 135619 & 25,05 & 149662 & 24,93 \\
Doenças do aparelho circulatório & 138163 & 25,52 & 144757 & 24,11 \\
Algumas doenças infecciosas e parasitárias & 125427 & 23,17 & 138526 & 23,07 \\
Neoplasias & 96761 & 17,87 & 101089 & 16,84 \\
Doenças do aparelho digestivo & 45394 & 8,39 & 66368 & 11,05 \\
\hline TOTAL & 541364 & 100,00 & 600402 & 100,00 \\
\hline
\end{tabular}

O quantitativo de óbitos por cor/raça registrados nos anos de 2016 a 2018, nas macrorregiões brasileiras aparece na tabela 3. Para as Regiões Norte, Nordeste e Centro-Oeste, o quantitativo de óbitos foi maior para a cor/raça parda enquanto que, para Sudeste e Sul, prevaleceu a branca.

Tabela 3. Quantitativo de óbitos por cor/raça registrados nos anos de 2016 a 2018, nas macrorregiões brasileiras.

\begin{tabular}{lllllll}
\hline & Branca & Preta & Parda & Amarela & Indígena & Sem informação \\
\hline Norte & 2044 & 828 & 34505 & 1062 & 378 & 18888 \\
Nordeste & 15170 & 5360 & 125906 & 8336 & 113 & 103357 \\
Centro-Oeste & 11071 & 1272 & 28805 & 1057 & 382 & 28326 \\
Sudeste & 246548 & 34004 & 146535 & 7201 & 75 & 103610 \\
Sul & 168199 & 6618 & 13638 & 1363 & 146 & 26969 \\
\hline TOTAL & 443032 & 48082 & 349389 & 19019 & 1094 & 281150 \\
\hline
\end{tabular}

A tabela 4 mostra o quantitativo de óbitos por faixa etária registrados nos anos de 2016 a 2018, nas macrorregiões brasileiras. Em relação à faixa etária, para todas as regiões o maior quantitativo de óbitos foi para maiores de 60 anos. 
Tabela 4. Quantitativo de óbitos por faixa etária registrados nos anos de 2016 a 2018, nas macrorregiões brasileiras.

\begin{tabular}{lllll}
\hline & 0 a 9 anos & 10 a 19 anos & 20 a 59 anos & $>60$ anos \\
\hline Norte & 3230 & 1038 & 18383 & 35317 \\
Nordeste & 7347 & 2932 & 70476 & 177487 \\
Centro-Oeste & 1680 & 695 & 20659 & 47879 \\
Sudeste & 7191 & 3428 & 136823 & 390531 \\
Sul & 2584 & 1321 & 52305 & 160723 \\
\hline TOTAL & 22032 & 9414 & 298646 & 811937 \\
\hline
\end{tabular}

A tabela 5 mostra o quantitativo de óbitos pelas principais causas, relacionadas à faixa etária, registrados nos anos de 2016 a 2018. De 0 a 9 e 10 a 19 anos, prevaleceram as causas infecciosas e parasitárias. De 20 a 59 anos, as neoplasias foram as principais causas. Por fim, para maiores de 60 anos, doenças do aparelho respiratório foram as mais comuns.

Tabela 5. Quantitativo de óbitos pelas principais causas, relacionadas à faixa etária, registrados nos anos de 2016 a 2018.

\begin{tabular}{|c|c|c|c|c|}
\hline & 0 a 9 anos & 10 a 19 anos & 20 a 59 anos & $>60$ anos \\
\hline Doenças do aparelho respiratório & 7888 & 2369 & 52541 & 223283 \\
\hline Doenças do aparelho circulatório & 1795 & 1303 & 63127 & 216695 \\
\hline Algumas doenças infecciosas e parasitárias & 8675 & 2787 & 71068 & 181423 \\
\hline Neoplasias & 1994 & 2216 & 72875 & 120765 \\
\hline Doenças do aparelho digestivo & 2480 & 739 & 38772 & 69771 \\
\hline TOTAL & 22832 & 9414 & 298383 & 811937 \\
\hline
\end{tabular}

\section{DISCUSSÃO}

O maior quantitativo de óbitos foi registrado na região Sudeste e o menor da região Norte (gráfico 1), resultados divergentes dos encontrados por Cervi, Hermsdorff e Ribeiro (2005), que demonstravam maior quantitativo para Sul e menor para o Nordeste. Esses resultados podem ser explicados pela transição epidemiológica, bem como pela mudança demográfica dos últimos anos, com maior concentração 
populacional no Sudeste e crescimento mais lento na região Norte, em detrimento às outras regiões (JACINTO, RIBEIRO, 2015; RIGOTTI, CAMPOS, HADAD, 2015).

Segundo Moura et al. (2016), o sexo masculino normalmente está relacionado a maior mortalidade devido a fatores como: especificidades biológico-genéticas; desigualdades e diferenças sociais; busca e uso de serviços de saúde por homens, cuidados de saúde voltados para homens; e diferentes expectativas sociais para ambos os sexos. No entanto, o presente estudo demonstrou maior número de óbitos para o sexo feminino, o que pode estar relacionado ao fato de a população feminina brasileira ser mais numerosa que a masculina (gráfico 2). Segundo a Pesquisa Nacional por Amostra de Domicílios Contínua (PNAD Contínua) de 2018, enquanto os homens representavam $48,3 \%$ da população residente, as mulheres correspondiam a $51,7 \%$. Além disso, os homens apresentaram padrão mais jovem que as mulheres (IBGE, 2019).

Em relação às causas de óbito, é necessário evidenciar que, apesar de as causas externas serem consideradas pela literatura como uma das principais que levam ao óbito no Brasil e no mundo, tomando lugar das causas transmissíveis, o mesmo não pode ser analisado em nosso estudo devido à carência de dados no DATASUS para essa causa. Sendo assim, as regiões Nordeste, Centro-Oeste e Sudeste apresentaram as doenças do aparelho circulatório como principal causa, enquanto que as regiões Norte e Sul, bem como o quantitativo brasileiro geral, evidenciaram as doenças do aparelho respiratório como causa principal (tabela 1). Por outro lado, segundo Mansur et al. (2009), desde 1980, vem sendo observada uma redução da mortalidade por doenças circulatórias, com queda maior nas regiões Sudeste e Sul e para as faixas etárias acima de 60 anos (CORRÊA, MIRANDA-RIBEIRO, 2017).

Ressalta-se, também, que o país enfrenta o desafio da coexistência de novos e velhos agravos, uma vez que a magnitude das doenças infecto-parasitárias continua elevada, além das grandes desigualdades regionais (OLIVEIRA-CAMPOS, CERQUEIRA, NETO, 2009). No entanto, é notável que a polarização entre os tipos de agravos à saúde em associação com a polarização geográfica deixou de persistir, já que as doenças infecto-parasitárias deixaram de ser causa principal de óbitos até mesmo nas 
regiões brasileiras consideradas menos desenvolvidas, ocupando a terceira posição (DE ARAÚJO, 2012).

O público feminino parece ser mais propenso ao desenvolvimento de problemas de saúde relacionados à circulação (tabela 2). Alguns estudos relacionam, por exemplo, a síndrome de ovários policísticos aos fatores que podem ocasionar problemas circulatórios (SOBRAL et al., 2019). Homens tendem a ter maiores complicações respiratórias devido ao comportamento social inerente ao gênero, visto que o hábito de fumar faz parte do grupo masculino (VIRTUOSO et al., 2010).

Ao analisar cor/raça, observou-se que grande quantidade de óbitos está sem esta informação disponível (tabela 3). Isto pode dar-se devido à difícil mensuração destes caracteres, já que possuem um nível de subjetividade maior do que o encontrado, por exemplo, na mensuração da idade ou do sexo dos entrevistados. Há variabilidade na autoclassificação étnica das pessoas e, como em outros países, também no Brasil a identidade racial não é fixa nem imutável e sua validade e confiabilidade são limitadas (ARAÚJO et al., 2009; CAMPOS, ESTANISLAU, 2016; CHOR, 2013). Até o Censo 2010, havia pouca informação sobre a mortalidade relacionada à cor/raça, sendo necessário, na época, aplicar um procedimento metodológico adicional para realizar a estimativa (CAMPOS et al., 2017; LOTUFO, 2015).

Considerando-se que, nas regiões Norte, Nordeste e Centro-Oeste do Brasil há mais concentração populacional de pardos e, nas Regiões Sudeste e Sul, prevalece a de brancos, o maior quantitativo de óbitos dessas cores/raças para os grupos de regiões citadas corresponde proporcionalmente aos padrões étnico-demográficos destes (tabela 3) (IBGE, 2019).

A maior concentração de óbitos em indivíduos a partir de 60 anos de idade (tabela 4) correspondeu ao descrito na literatura, já que o aumento da expectativa de vida da população atual também se associa a maior morbidade e mortalidade na velhice. A causa principal dos óbitos nesta faixa etária costuma ser por doenças circulatórias, porém, segundo Mansur et al. (2009), houve redução de tais doenças após os 60 anos, bem como nas áreas mais desenvolvidas do país. Além disso, Rosa et al. (2010) 
também demonstrou o crescimento das doenças do aparelho respiratório como causa de hospitalização e mortalidade para essa faixa etária, o que pode justificar a tendência atual de tais doenças serem principal causa de óbitos nos idosos brasileiros (tabela 5) (BALDONI, PEREIRA, 2011; MAIA et al., 2018; MOURA et al., 2015).

O Brasil é um país de renda média superior, e as doenças infecciosas não são causas tão dominantes de mortalidade em comparação aos países de baixa renda (AXELSSON, SILFVERDAL, 2011). No entanto, nosso estudo demonstrou que, para as faixas etárias de 0 a 9 e 10 a 19 anos, o impacto das doenças infecto-parasitárias ainda é importante (tabela 5), mesmo diante da transição epidemiológica, através da qual as doenças crônicas não transmissíveis tendem a ser mais prevalentes, devido à melhoria da renda da população menos favorecida, além das políticas de saúde e sociais, que tem facilitado o acesso da população aos serviços de saúde. As doenças infecciosas tendem a ter maior incidência em cidade maiores, onde a disseminação se faz de maneira mais veloz em ambientes mais povoados. Esse padrão pode explicar a persistência de tais índices em crianças e jovens, já que a proximidade em meio escolar propicia o contágio, este também potencializado pelo comportamento de risco em relação ao autocuidado e à exposição ambiental (JÚNIOR et al., 2017; SEGURADO, CASSENOTE, LUNA, 2016).

Outro fator de risco que deve ser levado em consideração está ligado a não vacinação de menores, seja por falha na cobertura vacinal ou por falta de adesão dos responsáveis, tornando esta faixa etária vulnerável a diversas doenças infecciosas preveníveis. A prática vacinal em massa é fundamentada na característica de imunidade de rebanho das vacinas, através da qual indivíduos imunes vacinados protegem indiretamente os não vacinados, de forma que a circulação do agente infeccioso no ambiente pode ser eliminada, com consequente proteção da coletividade e de indivíduos vulneráveis (BARBIERI, COURO, AITH, 2017).

Para a faixa etária de 20 a 59 anos, a predominância de neoplasias (tabela 5) pode ser explicado pelo aumento dos fatores de risco para este grupo de doenças, decorrente da transição epidemiológica. Alterações no padrão alimentar, por exemplo, tem papel importante na determinação do risco. Excessiva ingestão calórica associa- 
se com aumento de risco de câncer de pulmão, esôfago e cólon, este último também ligado ao aumento do consumo de alimentos gordurosos. Além disso, a redução no consumo de frutas e hortaliças também contribuem para o desenvolvimento de diversas neoplasias (CERVI, HERMSDORFF, RIBEIRO, 2005). Em 1998, as notificações por neoplasia no Brasil foram de 82.224 casos e, no ano de 2015, foram 278.960, significando um crescimento de $239,27 \%$ em 17 anos (VANZELLA, NASCIMENTO, SANTOS, 2018).

\section{CONCLUSÃO}

O perfil da mortalidade brasileira tem sido modificado pela transição epidemiológica, apresentando quantitativos maiores nas Regiões mais desenvolvidas e associação com as DCNT, principalmente as doenças do aparelho respiratório.

O sexo feminino foi o que apresentou maiores taxas de óbitos, tendo doenças do aparelho circulatório como a principal causa. Para o sexo masculino, prevaleceram as respiratórias.

O maior quantitativo de óbitos para pardos no Norte, Nordeste e Centro-Oeste, bem como de brancos para Sudeste e Sul corresponderam proporcionalmente ao padrão étnico das regiões. Evidenciou-se, no entanto, a grande quantidade de óbitos sem informação sobre cor/raça.

A maior mortalidade na faixa etária a partir de 60 anos correspondeu ao descrito na literatura, apesar das causas principais divergirem do padrão encontrado em outros estudos.

Fica clara a importância dos registros das informações sobre óbitos, bem como da melhoria na coleta de dados e sistematização destes, visto que possibilitam melhor execução de políticas públicas brasileiras para prevenção e cuidados em saúde, promovendo a dignidade de toda a população. 


\section{REFERÊNCIAS}

ALVES, C. G.; MORAIS NETO, O. L. Tendência da mortalidade prematura por doenças crônicas não transmissíveis nas unidades federadas brasileiras. Ciências e Saúde Coletiva, Rio de Janeiro, v. 20, n. 3, p. 641-654, 2015.

ANDRADE, M.V.; NORONHA, K.V.M.S.; MENEZES, R.M.; SOUZA, M.N.; REIS, C.B.; MARTINS, D.R.; GOMES, L. Desigualdade socioeconômica no acesso aos serviços de saúde no Brasil: um estudo comparativo entre as regiões brasileiras em 1998 e 2008. Economia Aplicada, Ribeirão Preto, v. 17, n. 4, p. 623-645, 2013.

ARAÚJO, E. M.; COSTA, M. C. N.; HOGAN, V. K.; ARAÚJO, T. M.; DIAS, A. B.; OLIVEIRA, L. O. A. A utilização da variável raça/cor em Saúde Pública: possibilidades e limite. Comunicação Saúde Educação, Botucatu, v.13, n.31, p.383-94, 2009.

AXELSSON, I.; SILFVERDAL, S. A. Mortalidade por pneumonia entre crianças brasileiras: uma história de sucesso. Jornal de Pediatria, Porto Alegre, v. 87, n. 2, p. 85-87, 2011.

BALDONI, A. O.; PEREIRA, L. R. L. O impacto do envelhecimento populacional brasileiro para o sistema de saúde sob a óptica da farmacoepidemiologia: uma revisão narrativa. Rev Ciênc Farm Básica Apl, Ribeirão Preto, v. 32, n. 3, p. 313-321, 2011.

BARBIERI, C. L. A.; COURO, M. T.; AITH, F. M. A. A (não) vacinação infantil entre a cultura e a lei: os significados atribuídos por casais de camadas médias de São Paulo, Brasil. Cad. Saúde Pública, Rio de Janeiro, v. 33, n. 2, e00173315, 2017.

BATISTA, J.; BARRETO, M.S.; MERINO, M.F.G.L.; FRACASSO, N.V.; BALDISSERA, V.D.A. Perfil epidemiológico da mortalidade por causas externas entre beneficiários de planos de saúde no Brasil. Revista de Enfermagem do Centro-Oeste Mineiro, 2018. Revista de Enfermagem do Centro-Oeste Mineiro, Divinópolis, v. 8, n. 1870, 2018.

CAMPOS, M. B.; BORGES, G. M.; QUEIROZ, B. L.; SANTOS, R. V. Diferenciais de mortalidade entre indígenas e não indígenas no Brasil com base no Censo 
Demográfico de 2010. Cad. Saúde Pública, Rio de Janaeiro, v. 33, n. 5, e00015017, 2017.

CAMPOS, M. B.; ESTANISLAU, B. R. Demografia dos povos indígenas: os Censos Demográficos como ponto de vista. R Bras Est Pop, Rio de Janeiro, v.33, n.2, p. 441449, 2016.

CERVI A.; HERMSDORFF, H. H. M.; RIBEIRO, R. C. L. Tendência da mortalidade pordoenças neoplásicas em 10capitais brasileiras, de 1980 a 2000. Rev Bras Epidemiol, São Paulo, v. 8, n. 4, p. 407-418, 2005.

CHOR, D. Desigualdades em saúde no Brasil: é preciso ter raça. Cad Saúde Pública, Rio de Janeiro, v. 29, n. 7, p. 1272-1275, 2013.

CORREA, E. R. P.; MIRANDA-RIBEIRO, A. Ganhos em expectativa de vida ao nascer no Brasil nos anos 2000: impacto das variações da mortalidade por idade e causas de morte. Ciência e Saúde Coletiva, Rio de Janeiro, v. 22, n. 3, p. 1005-1015, 2017.

DE ARAÚJO, J. D. Polarização epidemiológica no Brasil. Epidemiologia e Serviços de Saúde, Brasília, v. 21, n. 4, p. 533-538, 2012.

FRANÇA, E. B.; CUNHA, C. C.; VASCONCELOS, A. M. N.; ESCALANTE, J. J. C.; ABREU, D. X.; LIMA, R. B.; NETO, O. L. M. Avaliação da implantação do programa "Redução do percentual de óbitos por causas mal definidas" em um estado do Nordeste do Brasil. Rev Bras Epidemiol, São Paulo, v. 17, n. 1, p. 119-134, 2014.

IBGE- Instituto Brasileiro de Geografia e Estatística. Características gerais dos domicílios e dos moradores 2018. Rio de Janeiro, 2019. Disponível em: https://loja.ibge.gov.br/pnad-continua-caracteristicas-gerais-dos-domicilios-e-dosmoradores-2018.html. Acesso em: 02/07/2019.

ISHTANI, L. H.; TEIXEIRA, R. A.; ABREU, D. M. X.; PAIXÃO, L. M. M. M.; FRANÇA, L. B. Qualidade da informação das estatísticas de mortalidade: códigos 
garbagedeclarados comocausas de morte em Belo Horizonte, 2011-2013. Rev Bras Epidemiol, São Paulo, v. 20, sUppl 1, p. 34-45, 2017.

JACINTO, P. A.; RIBEIRO, E. P. Crescimento e envelhecimento populacional brasileiro: menos trabalhadores e trabalhadores mais produtivos? Pesquisa e Planejamento Econômico, Brasília, v. 45, n. 2, p. 177-217, 2015.

JÚNIOR, A. F. C.; MATOS, E. C. O.; FILOCREÃO, B. L.; SILVA, C. C.; SOARES, M. I. S.; COSTA, R. C. L. Riscos infecciosos no ambiente escolar: relato de experiência com escolares através de metodologia ativa. Sau. \& Transf. Soc., Florianópolis, v. 8, n. 2, p.128-134, 2017.

LAURENTI, R.; DI NUBILA, H.B.V.; QUADROS, A.A.J.; CONDE, M.T.R.P.; OLIVEIRA, A.S.B. A classificação Internacional de Doenças Internacionais, a CID-11 e a Síndrome Pós-Poliomielite. Arquivos de Neuro-Psiquiatria, São Paulo, v. 71, n. 9, p. 3-10, 2013.

LEITE, I.C.; VALENTE, J.G.; SCHRAMM, J.M.A.; DAUMAS, R.P.; RODRIGUES, R.N.; SANTOS, M.F.; DE OLIVEIRA, A. F.; DA SILVA, R. S.; CAMPOS, M. R.; DA MOTA, J. C. Carga de doença no Brasil e suas regiões, 2008. Caderno de Saúde Pública, Rio de Janeiro, v. 31, n. 7, p. 1551-1564, 2015.

LOTUFO, P. A. Ethnicity and cardiovascular mortality in Brazil: a call for papers. Sao Paulo Med J, São Paulo, v. 133, n. 3, p. 169-70, 2015.

MAIA, C. S.; FREITAS, D. R. C.; GALLO, L. G.; ARAÚJO, W. N. Notificações de eventos adversos relacionados com a assistência à saúde que levaram a óbitos no Brasil, 2014-2016. Epidemiol Serv Saude, Brasília, v. 27, n. 2, e2017320, 2018.

MANSUR, A. P.; LOPES, A. I. A.; FAVARATO D., AVAKIAN S. D.; CÉSAR, L. A. M.; RAMIRES, J. A. F. Transição Epidemiológica da mortalidade por doenças Circulatórias no Brasil. Arq Bras Cardiol, Rio de Janeiro, v. 83, n. 5, p. 506-510, 2009. 
MARINHO, F.; PASSOS, V.M.A.; FRANÇA, E.B. Novo século, novos desafios: mudança no perfil da carga de doença no Brasil de 1990 a 2010. Epidemiologia e Serviços de Saúde, Brasília, v. 25, n. 4, p. 713-724, 2016.

MARTINS-MELO, F.R.; JUNIOR, A. N. R.; ALENCAR, C. H.; HEUKELBACH, J. Multiple causes of death related to Chagas' disease in Brazil, 1999 to 2007. Revista da Sociedade Brasileira de Medicina Tropical, Uberaba, v. 45, n. 5, p. 591-596, 2012.

MARTINS, R. C.; BUCHALLA, C. M. Codificação e seleção automáticas das causas de morte: adaptação para o uso no Brasil do software Iris. Rev Bras Epidemiol, São Paulo, v. 18, n. 4, p. 883-893, 2015.

MOURA, E. C.; GOMES, R.; FALCÃO, M. T. C.; SCHWARZ, E.; NEVES, A. C. M.; SANTOS, W. Desigualdades de gênero na mortalidade por causas externas no Brasil, 2010. Ciência \& Saúde Coletiva, Rio de Janeiro, v. 20, n. 3, p. 779-788, 2015.

MOURA, E. C.; SANTOS, W.; NEVES, A. C. M.; SCHWARZ, E.; GOMES, R. Mortalidade no Brasil segundo perspectiva de gênero, anos 2000 e 2010. Rev Bras Epidemiol, Rio de Janeiro, v. 19, n. 2, p. 326-338, 2016.

OLIVEIRA-CAMPOS, M.; CERQUEIRA, M. B. R.; NETO, J. F. R. Dinâmica populacional e o perfil de mortalidade no município de Montes Claros (MG). Ciência \& Saúde Coletiva, Rio de Janeiro, v. 16, Supl. 1, p. 1303-1310, 2011.

PAES, N.A.; GOUVEIA, J. Recuperação das principais causas de morte do Nordeste do Brasil: impacto na expectativa de vida. Revista de Saúde Pública, São Paulo, v. 44, n. 2, p. 301-309, Abr. 2009.

RIGOTTI, J. I. R.; CAMPOS, J.; HADAD, R. M. Migrações internas no Brasil: (des)continuidades regionais à luz do Censo Demográfico 2010. Blucher Social Sciences Proceedings, São Paulo, v. 2, n. 2, p. 29-46, 2015. 
ROSA, L. H. T.; ROSSATO, T. T.; BOMBARDELLI, C. L.; STURMER, C. L.; ROSA, P. V. Estudo da mortalidade em população idosa de municipios do RioGrande do Sul no período de 1996 a 2004. Rev Bras Geriatr Gerontol, Rio de Janeiro, v. 13, n. 1, p. 111-119, 2010.

SEGURADO, A.C.; CASSENOTE, A.J.; LUNA, E.A. Saúde nas metrópoles - Doenças infecciosas. Estudos Avançados, São Paulo, v. 30, n. 86, p. 29-49, 2016.

SIQUEIRA, A.S.E.; SIQUEIRA-FILHO, A.G.; LAND, M.G. P. Análise do impacto econômico das doenças cardiovasculares nos últimos cinco anos no Brasil. Arquivo Brasileiro de Cardiologia, São Paulo, v. 109, n. 1, p. 39-46, 2017.

SOBRAL, J.P.C.P.; SANTOS, J.M.S.; ARAUJO, R.J.S.; SANTOS, T.G. A Mulher e as Doenças Cardiovasculares: Morbidade Hospitalar em Alagoas. GEPNEWS, v.1, n.1, p.32-38, 2019

SOUZA, M.F.M.; MALTA, D.C.; FRANÇA, E.B.; BARRETO, M.L. Transição da saúde e da doença no Brasil e nas Unidades Federadas durante os 30 anos do Sistema Único de Saúde. Ciência e Saúde Coletiva, Rio de Janeiro, v. 23, n. 6, p. 17371750, 2018.

VANZELLA, E.; NASCIMENTO, J. A.; SANTOS, S. R. O envelhecimento, a transição epidemiológica da população brasileira e o impacto nas hospitalizações. Rev Elet Estácio Saúde, Santa Catarina, v. 7, n. 1, p. 65-73, 2018.

VIRTUOSO, J.F.; BALBÉ, G.P.; MAZO, G.Z.; PEREIRA, M.G.S.; SANTOS, F.S. Morbidade e mortalidade da população idosa de Florianópolis: um estudo comparativo entre homens e mulheres. Rev. Bras. Geriatr. Gerontol. v.13, n. 2, p. 215-223, 2010.

Enviado: Setembro, 2019.

Aprovado: Outubro, 2019. 